\title{
The promoter of Bmlp3 gene can direct fat body-specific expression in the transgenic silkworm, Bombyx mori
}

\author{
DangJun Deng $\cdot$ HanFu Xu $\cdot$ Feng Wang • \\ Xiaoli Duan · SanYuan Ma $\cdot$ ZhongHuai Xiang • \\ QingYou Xia
}

Received: 14 January 2013/Accepted: 19 March 2013/Published online: 30 March 2013

(C) The Author(s) 2013. This article is published with open access at Springerlink.com

\begin{abstract}
The fat body plays multiple, crucial roles in the life of silkworms. Targeted expression of transgenes in the fat body of the silkworm, Bombyx mori, is important not only for clarifying the function of endogenous genes expressed in this tissue, but also for producing valuable recombinant proteins. However, fat body-specific gene expression remains difficult due to a lack of suitable tissue-specific promoters. Here we report the isolation of the fat body-specific promoter of Bmlp3, a member of the $30 \mathrm{~K}$ protein family of silkworms. The $1.1 \mathrm{~kb}$ fragment from -374 to +738 of Bmlp3 displayed strong promoter activity in the cell lines BmE and Spli-221. In transgenic silkworms, a DsRed reporter gene controlled by the
\end{abstract}

DangJun Deng and HanFu Xu contributed equally to this work.

Electronic supplementary material The online version of this article (doi:10.1007/s11248-013-9705-8) contains supplementary material, which is available to authorized users.

D. Deng $\cdot$ H. Xu $\cdot$ F. Wang $\cdot$ X. Duan .

S. Ma $\cdot$ Z. Xiang $\cdot$ Q. Xia $(\bowtie)$

State Key Laboratory of Silkworm Genome Biology,

Southwest University, Chongqing 400715,

People's Republic of China

e-mail: xiaqy@swu.edu.cn

D. Deng

Institute of Forensic Science, Chongqing Public Security

Bureau, Chongqing 401147, People's Republic of China
$1.1 \mathrm{~kb}$ Bmlp3 promoter fragment was expressed specifically in the fat body in a stage-specific pattern that was nearly identical to the endogenous Bmlp3 gene. We conclude that the $1.1 \mathrm{~kb} \mathrm{Bmlp3}$ promoter fragment is sufficient to direct tissue- and stagespecific expression of transgenes in the fat body of silkworms, highlighting the potential use of this promoter for both functional genomics research and biotechnology applications.

Keywords Bmlp3 promoter - Fat body - Specific expression · Transgene $\cdot$ Silkworm

\section{Introduction}

The fat body of the silkworm, Bombyx mori, is a relatively large tissue distributed throughout the body. As the central storage tissue for nutrients and energy reserves, the fat body plays crucial roles in the life of silkworms including development, metamorphosis, immunity and reproduction. Large amounts of storage proteins, lipoproteins and vitellogenins are synthesized in the fat body, many of which are released and accumulated in a sex- and stage-specific manner in the hemolymph. Consequently, the fat body represents an ideal model tissue for studying metamorphosis and the developmental regulation of gene expression (Izumi et al. 1981; Sakurai et al. 1988a, b; Sakai et al. 1988). Recently, the analysis of wholegenome DNA sequence data, large-scale expressed sequence tag data and microarray-based gene expression 
profiles revealed numerous tissue-specific genes responsible for the accurate regulation and prodigious biosynthesis of proteins in the fat body of B. mori (Xia et al. 2004, 2007; Cheng et al. 2006). These findings provide a unique opportunity to better understanding the elusive biological roles of the fat body by clarifying the function of these genes.

Analyzing gene function is greatly aided by transgenic technology in which gene expression is regulated temporally and spatially. Since the first successful germline transformation of $\mathrm{B}$. mori using the piggyBac transposon (Tamura et al. 2000), dozens of genes have been studied using transgenic methods alone or in combination with other genetic tools. In many cases, as reported in other model organisms, the use of tissueand/or stage-specific promoters has proven to be very important. Unfortunately, it has been difficult to study the fat body of the silkworm using this strategy because of the lack of suitably specific promoters. Moreover, considering the extraordinary ability of the fat body of the silkworm to synthesize and store large amounts of proteins, this tissue might be developed into a novel bioreactor to produce valuable recombinant proteins. To test these ideas it is necessary to isolate fat body-specific promoters that lead to high levels of gene expression in useful temporal and spatial patterns.

Among the major proteins synthesized by the fat body of B. mori are a group of low molecular weight lipoproteins (Bmlps) with molecular weights around $30 \mathrm{kDa}$, and known as " $30 \mathrm{~K}$ proteins". $30 \mathrm{~K}$ proteins, are synthesized in a specific temporal patterns and released into the hemolymph during the post-embryonic development (Tojo et al. 1980; Izumi et al. 1981,1984; Tomino 1985). So far, a total of 24 genes encoding typical 30K proteins (Bmlpl-24) have been identified in the genome of B. mori (Zhang et al. 2012a, b). Interestingly, at least six of them, including Bmlpl-4, Bmlp 7 and Bmlp9, were abundantly expressed in the fat body in a developmentally regulated pattern (Sun et al. 2007; Hou et al. 2007; Zhang et al. 2012a, b), suggesting that the regulatory regions of these genes might be useful for controlling the expression of transgenes in the fat body of silkworms.

The objective of this study was to isolate the $5^{\prime}$ regulatory region of $B m l p 3$ and to test whether it can direct fat body-specific expression of transgenes in the silkworm. We isolated the $5^{\prime}$ regulatory region of Bmlp3 and tested its activity in insect cell lines using a Dual-luciferase reporter assay, and transgenic silkworms by fusing a Bmlp3 promoter-containing fragment to a $D s R e d$ reporter gene. The results showed tissue- and stage-specific expression of DsRed in the fat body of transgenic silkworms, demonstrating the utility of the Bmlp3 promoter for both functional genomic studies and biotechnology applications.

\section{Materials and methods}

\section{Silkworm strain}

The silkworm strain P50 was maintained in our laboratory and used for promoter isolation and germline transformation. Eggs were maintained at $25^{\circ} \mathrm{C}$ with $95 \%$ humidity until hatching, and larvae were reared on fresh mulberry leaves at $25^{\circ} \mathrm{C}$.

Vector construction

The 1,119 bp promoter region (from -374 to +745 ) upstream of the Bmlp3 gene was amplified using the primers Bmlp3-PF1/Bmlp3-PR1 (Table S1) and cloned into pMD19-T simple vector (TaKaRa). To investigate the activity of this promoter, the DNA fragment was cut with $S m a I$ and $X h o I$ and inserted into the pGL3-basic vector (Promega) which contained the luciferase reporter gene, to generate the transient expression vector Bmlp3-pGL3.

To construct the transgenic vector pBacBmlp3DsRed/3 $\times$ P3EGFP, the Bmlp3 promoter region was amplified from the plasmid Bmlp3-pGL3 using the primers Bmlp3-PF2/Bmlp3-PR2 (Table S1), and assembled in the shuttle vector pSLfa1180fa (Horn and Wimmer 2000) by fusing in turn with DsRed and SV40 polyA signal. Then the Bmlp3-DsRed-SV40 cassette was cloned into the unique $A s c \mathrm{I}$ site of the piggyBac-containing vector $\mathrm{pBac}[3 \times \mathrm{P} 3 \mathrm{EGFPafm}]$ (Horn and Wimmer 2000) to generate the final donor vector.

Cell culture and dual-luciferase reporter assay

The B. mori embryonic cell line BmE and ovarian cell line $\mathrm{BmN}$, the Spodoptera frugiperda ovarian cell lines Sf9 and Sf21, and the Spodoptera litura embryonic cell line Spli-221, were maintained at $27{ }^{\circ} \mathrm{C}$ in 
Grace's media supplemented with $10 \%$ fetal bovine serum (FBS, Hyclone, China), $50 \mathrm{U} / \mathrm{mL}$ penicillin and $50 \mathrm{mg} / \mathrm{mL}$ streptomycin. For transfections, cells were seeded onto a 24 -well tissue culture plate $\left(1 \times 10^{5}\right.$ cells/well) for $12 \mathrm{~h}$. One hundred microliters of a mixture containing $1 \mu \mathrm{g}$ Bmlp3-pGL3 plasmid DNA, $0.1 \mu \mathrm{g}$ pRL-SV40 plasmid DNA $3 \mu \mathrm{l}$ LipofectAMINE 2000 (Invitrogen) was incubated in the serum-free Grace's media with BmE, BmN, Sf9, Sf21 or Spli-221 cells for 4-6 h. Plasmid pRL-SV40 (Promega) is a $3.7 \mathrm{~kb}$ plasmid with the luciferase gene from Renilla reniformis under the regulatory control of the SV40 promoter. The transfection mixture was replaced with $500 \mu \mathrm{L}$ fresh medium containing $10 \%$ FBS and incubated for an additional $24 \mathrm{~h}$. The transfected cells were washed twice with $1 \times$ phosphate-buffered saline (PBS) and lysed by $100 \mu \mathrm{L} /$ well lysis Buffer (Promega), the supernatant of cell lysis were further collected by centrifugating at 12,000 rpm, under $4{ }^{\circ} \mathrm{C}$ for $15 \mathrm{~min}$. Dual-Luciferase reporter assays were performed on Modulus ${ }^{\mathrm{TM}}$ single tube multimode reader (Promega) according to the manufacturer's protocols. Luciferase activity from expression of Bmlp3-pGL3 was normalized to luciferase activity from pRL-SV40. All experiments were repeated three times independently and the results reported as mean $\pm \mathrm{SE}$.

Germline transformation and image analysis

Transgenic B. mori were created using previously described methods (Tamura et al. 2000). Plasmids pBacBmlp3-DsRed/3 $\times$ P3EGFP and pHA3PIG (a piggyBac transposase-expressing 'helper' plasmid) were purified using QIAGEN Plasmid Midi Kit (Qiagen). Purified plasmid DNA was dissolved in super-pure water. The mixture of the donor and helper DNA, 400 and $400 \mu \mathrm{g} / \mu \mathrm{L}$, respectively, was injected into the pre-blastoderm eggs $1-2 \mathrm{~h}$ after oviposition. G0 moths developing from injected embryos were mated with each other to generate G1 progeny. Day-7 G1 embryos were screened for EGFP expression in the ocelli and compound eyes using an Olympus MVX10 fluorescent stereomicroscope (Olympus, Japan) equipped with appropriate filters for the detection of EGFP and RFP fluorescence. EGFP-positive individuals were reared to adults and siblings were mated to generate G2 offspring. DsRed expression in the dissected fat bodies of G2 larva were observed using a fluorescent stereomicroscope as described above.

Molecular analysis

\section{Southern blotting}

Genomic DNA from transgenic and wild-type moths was extracted using an improved phenol/chloroform method (Zhao et al. 2010). DNA samples $(10 \mu \mathrm{g})$ were fully digested using the restriction endonucleases HindIII and XhoI. The resulting DNA fragments were size fractionasted by gel electrophoresis in $1 \%(\mathrm{w} / \mathrm{v})$ agarose. Size-fractionated DNA was stained in-gel with ethidium bromide, or transferred directly to nylon membranes (Roche Applied Science) using vacuum transfer methods. DNA-containing nylon membranes were hybridized with a digoxigenin-labeled probe specific for the EGFP open reading frame (Table S1) at $65{ }^{\circ} \mathrm{C}$. Hybridized digoxigenin-labeled probes were detected using chemiluminescent methods with CDPStar (Roche, USA) according to the manufacturer's instructions.

\section{Inverse PCR}

Inverse PCR experiments were performed to identify the genomic location of integrated piggyBac vectors using genomic DNA purified from transgenic G1 moths and primers (Table S1) specific to the $5^{\prime}$ and $3^{\prime}$ ends of the piggyBac transposon as described (Tamura et al. 2000). The inverse PCR products were cloned into the vector pMD19-T (TaKaRa) and their DNA sequences determined.

\section{$R T-P C R$}

Total RNA from transgenic and wild-type larvae was isolated using the TRIzol reagent (Invitrogen). Isolated RNA ( $2 \mathrm{mg}$ ) was treated with DNaseI to remove trace amounts of genomic DNA and then used as templates for the synthesis of DNA. cDNA was synthesized using the Reverse Transcriptase M-MLV Kit (TaKaRa) according to the manufacturer's protocol. The PCR reactions were performed as follows using gene-specific primers (Table $\mathrm{S} 1$ ): denaturation at $94{ }^{\circ} \mathrm{C}$ for $3 \mathrm{~min}, 26$ cycles of $94{ }^{\circ} \mathrm{C}$ for $30 \mathrm{~s}, 55^{\circ} \mathrm{C}$ 
A

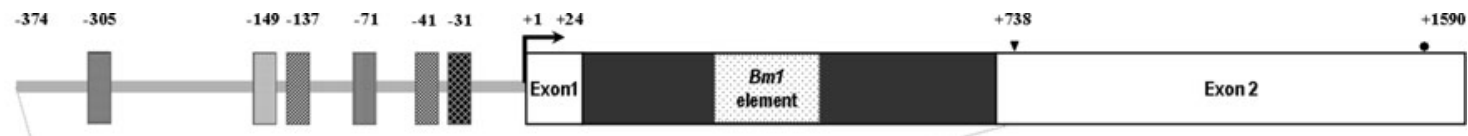

B

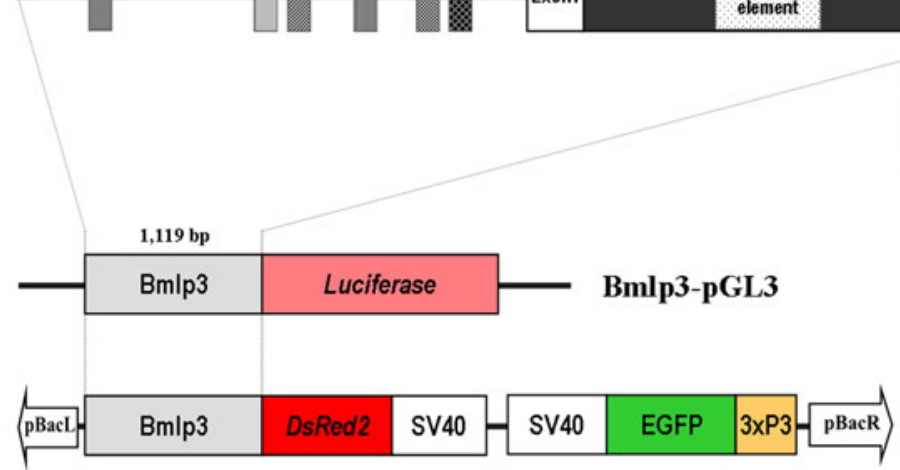

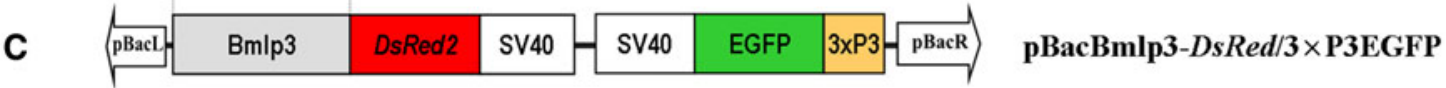

Fig. 1 Schematic diagram of the Bmlp3 promoter and two vectors. a Genomic organization of Bmlp3. The predicted transcription start site is denoted +1 . Exons are shown as white rectangles and intron is shown as black rectangles. The initiation codon (ATG) and stop codon (TAA) are indicated by a triangle and star respectively in exon2. The TATA box, CAAT box, and various putative motifs are shown as differently

for $30 \mathrm{~s}$ and $72{ }^{\circ} \mathrm{C}$ for $45 \mathrm{~s}$. The PCR products were separated on $1 \%$ agarose gels and visualized by staining with ethidium bromide.

\section{Western blotting}

Proteins from transgenic and wild-type day-7 fifth instar larvae were extracted with $1 \times$ PBS, incubation at room temperature overnight followed by centrifugation at $10,000 \mathrm{rpm}$ in a microcentrifuge for $5 \mathrm{~min}$. Samples of the supernatants were used to estimate the protein concentration by the Bradford protein assay and part was stored at $-20{ }^{\circ} \mathrm{C}$. Each sample of $10 \mu \mathrm{g}$ protein was subjected to SDS-PAGE and transferred to a poly (vinylidene difluoride) membrane. RFP was detected using an anti-RFP antibody (Gene. Co) and His-tagged RFP (BaiRui. Co) was used as a positive control. Quantity of RFP is determined by densitometric measurement of the immunoblot using a free Quantity One software(BioRad).

\section{Results and discussion}

Isolation and activity detection of the Bmlp3 promoter

Basing on bioinformatic analyses, a 1,119 bp genomic DNA fragment upstream of the Bmlp3 gene was shaded boxes as indicated in the figure. Numbers above the exon and cis-element boxes denotes the position of nucleotides in the gene relative to the transcription start site. b Schematic diagram of the transient expression vector Bmlp3-pGL3. c Schematic diagram of the transgenic expression vector $\mathrm{pBacBmlp3}$-DsRed/ $3 \times$ P3EGFP

identified as putatively containing the promoter and isolated by PCR from the silkworm strain P50. The fragment comprised 24 bp of exon1, 713 bp of intron1, $8 \mathrm{bp}$ of partial sequence of exon2 ( 9 bp distance from the initiation codon), and $374 \mathrm{bp}$ of $5^{\prime}$-flanking region upstream of transcription initiation site. Besides the core promoter sequences including the TATA-box and CAAT-box, several conserved motifs reported previously were detected including Spl-binding and Pbx-1 consensus sequences, an octamer-like sequence, a Bml element and a common sequence found in storage protein genes (Mori et al. 1991a, b; Sakurai et al. 1988a, b; Willott et al. 1989; Matsumoto et al. 1986; Delaney et al. 1986; Fujiwara and Yamashita 1992; Ogawa et al. 2005) (Fig. 1a).

To examine the activity of the Bmlp3 promoter, a quantitative assessment of luciferase activity was measured in five insect cell lines, BmE, BmN, Sf9, Sf21 and Spli-221, using a Dual-Luciferase reporter assay. The results showed that the Bmlp3 promoter had highly activity in BmE and Spli-221 cells, with the activity in Spli-221 was higher than in BmE (Figs. 1b, 2). However, no activity was detected in BmN, Sf9 and Sf 21 cells (data not shown), and the reason might be the lack of essential regulatory factors responsible for the activity of Bmlp3 promoter in these cell lines. Taken together, these results suggest that the $1,119 \mathrm{bp}$ fragment from the $5^{\prime}$ region of $B m l p 3$ has promoter activity under some conditions and might be sufficient 


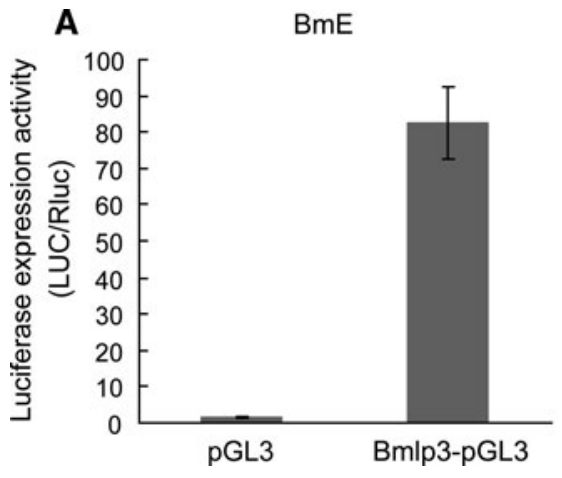

Fig. 2 Promoter activity of the 1,119 bp fragment from the $5^{\prime}$ region of Bmlp3 in insect cell lines. Expression of luciferase derived from Bmlp3-pGL3 in BmE (a) and Spli-221(b) cell lines. The activity of luciferase was measured at $72 \mathrm{~h}$ after co-

to direct expression of transgenes in the fat body of silkworm.

Generation of the transgenic silkworm

To test whether the putative 1,119 bp Bmlp3 promoter-containing fragment can direct fat body-specific expression in silkworms, we fused the fragment to a DsRed reporter gene (Fig. 1c) and then introduced the construct into the genome of B. mori. From 357 injected silkworm embryos a total of 126 moths were recovered and crossed, leading to 5 broods that produced EGFP-positive progeny. Transgenic G1 moths were randomly selected and genomic DNA was isolated and used to check for the presence of integrated transgene-containing piggyBac vectors by Southern blotting and inverse PCR analysis using an EGFP-specific probe and piggyBac-specific primers, respectively. The results indicated that the transgenic silkworms harbored single insertion located in Chromosome 20 (Fig. S1), suggesting only one transposition event during germ-line transformation. This transgenic line harboring single copy of the DsRed reporter gene was used for subsequent expression analyses.

Fluorescent detection of DsRed expression in transgenic silkworm

As shown in Fig. 3, pBacBmlp3-DsRed/ $3 \times$ P3EGFP resulted in high levels of RFP

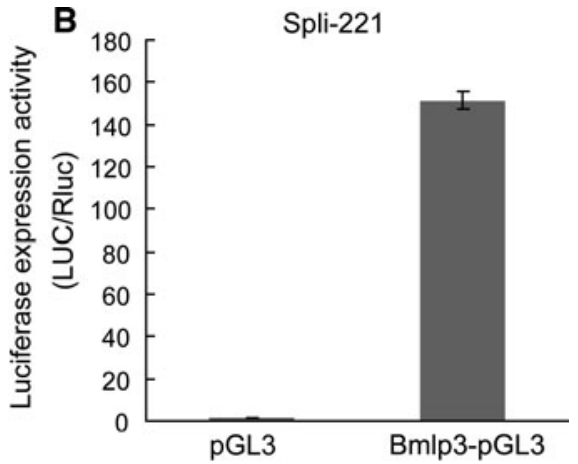

transfection with Bmlp3-pGL3 and pRL-SV40. The results were calculated from three independent experiments and described as the mean ratio of Firefly luciferase (from Bmlp3-pGL3) to Renilla luciferase (from pRL-SV40) \pm standard deviation

fluorescence in transgenic progeny. RFP fluorescence could not be detected in early developmental stages until day-4 of the fifth instar of transgenic silkworm. The intensity of RFP fluorescence increased with time during larval development and could be observed clearly in the body (Fig. 3a). During the pupal stage, a bright and intense RFP fluorescence was detected in whole body, indicating the high level expression of DsRed in this stage (Fig. 3b). DsRed expression continued throughout the pupal stage but decreased in adults with no obvious differences between expression in males and females (Fig. 3c). Dissected day-7 fifth instar larvae had RFP fluorescence localized entirely within the fat body, which was distributed throughout the insect (Fig. 3d, e). No RFP fluorescence was detected in other organs including the silk gland, gonads, midgut and hemolymph (Fig. 3f). These results show that the 1,119 bp fragment from the $5^{\prime}$ region of Bmlp3 has fat-body specific promoter activity.

Spatial and temporal expression of DsRed directed by the Bmlp3 promoter

To further investigate the expression patterns of $D$ sRed controlled by the Bmlp3 promoter, mRNA was extracted from different tissues of day-7 fifth instar larvae and the fat body from different developmental stages including day-1 fourth instar larvae to adults and analyzed by RT-PCR and Western blotting. As shown in Fig. 4, strong expression of Bmlp3-regulated DsRed 


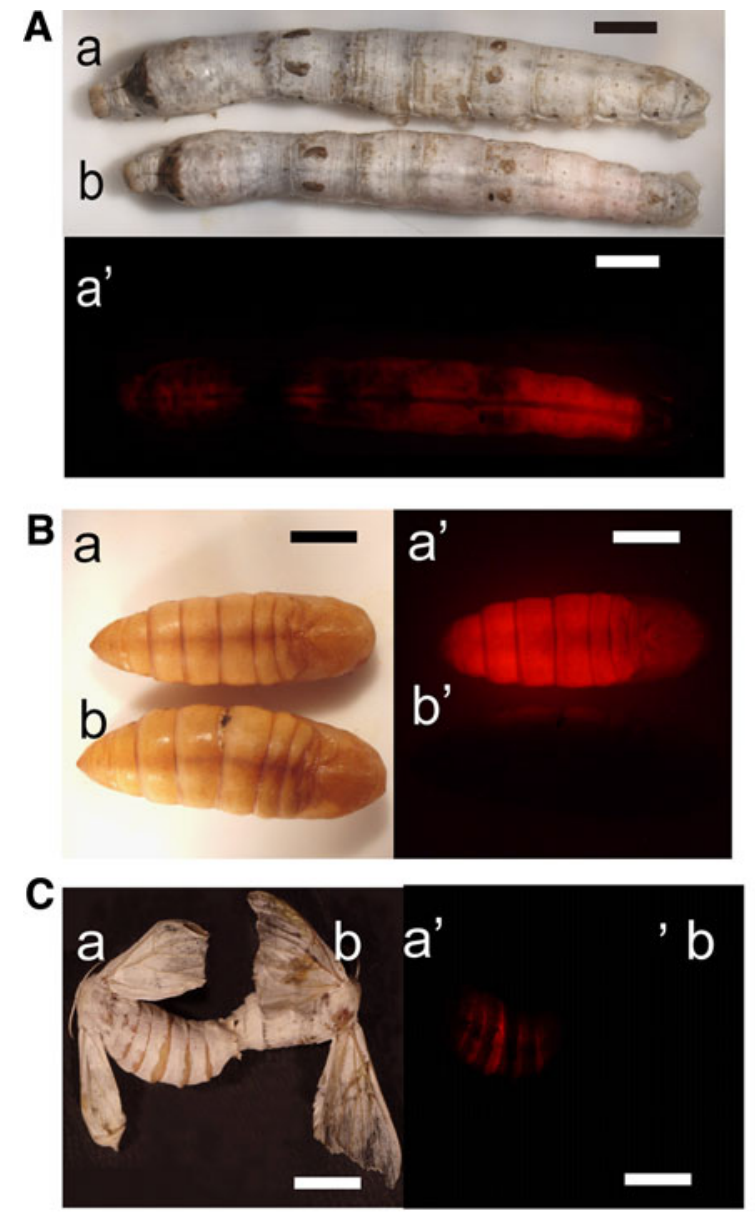

Fig. 3 Fluorescent microscopic detection of DsRed expression in transgenic silkworm. a Fluorescent detection of DsRed in the transgenic day-4 fifth instar larvae. Wild type $\left(a, a^{\prime}\right)$ and transgenic silkworm $\left(b, b^{\prime}\right)$ were viewed under white light and $565 \mathrm{~nm}$ ultraviolet light (optimal for detecting RFP fluorescence). b Fluorescent detection of DsRed in the transgenic pupae. Transgenic $\left(a, a^{\prime}\right)$ and wild type pupae $\left(b, b^{\prime}\right)$ were viewed under white light and $565 \mathrm{~nm}$ ultraviolet light. c Fluorescent detection of DsRed in the transgenic adults. Transgenic $\left(a, a^{\prime}\right)$ and wild type adults $\left(b, b^{\prime}\right)$ were viewed under white light and $565 \mathrm{~nm}$ ultraviolet light. d Fluorescent detection of DsRed in dissected transgenic day-7 fifth instar larvae.

was detected in the fat body of transgenic silkworms, and this was consistent with the pattern of expression of the endogenous Bmlp3 gene. Unexpectedly, low levels of DsRed transcripts were detected in the silk glands of transgenic silkworms, however DsRed
D

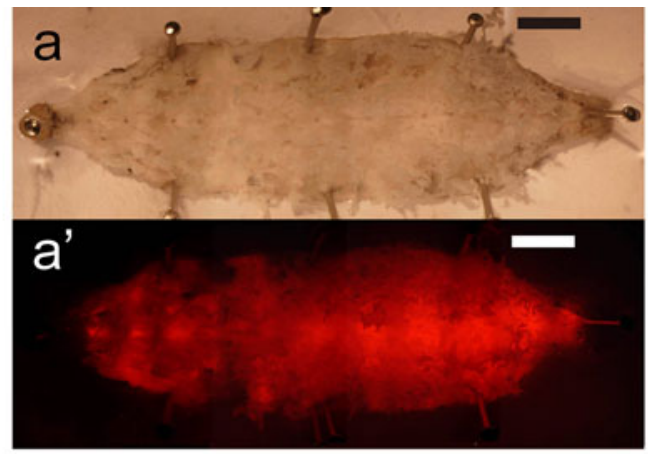

$\mathbf{E}$

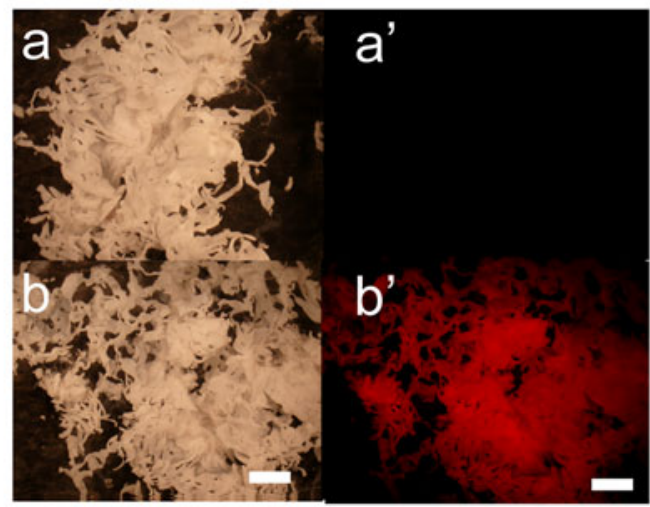

$\mathbf{F}$

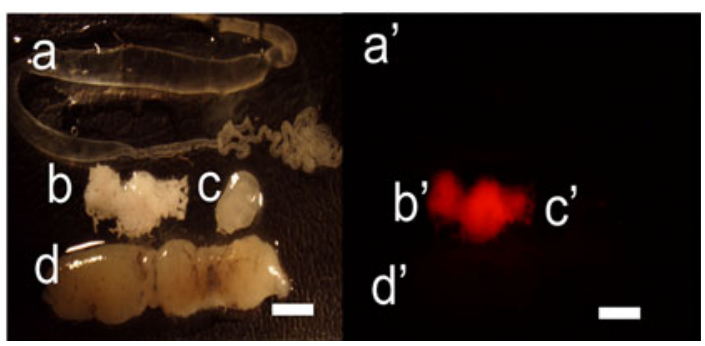

Transgenic larvae was viewed under white light $(a)$ and $565 \mathrm{~nm}$ ultraviolet light $\left(a^{\prime}\right)$. e DsRed fluorescent detection the isolated fat body from transgenic and wild-type day-7 fifth instar larvae. Fat body of wild type $\left(a^{\prime}\right)$ and transgenic larvae $\left(b, b^{\prime}\right)$ were dissected in $1 \times$ PBS and viewed under white light or $565 \mathrm{~nm}$ ultraviolet light. f Tissue-specific fluorescent detection of DsRed in the transgenic day-7 fifth instar larvae. Various tissues including the silk glands $\left(a^{\prime}\right)$, fat body $\left(b, b^{\prime}\right)$, gonads $\left(c, c^{\prime}\right)$ and midgut $\left(d, d^{\prime}\right)$ from transgenic larvae were viewed under white light and $565 \mathrm{~nm}$ ultraviolet light. The scale bar represents $0.5 \mathrm{~cm}$

protein was undetectable in this tissue. We speculate that regulatory elements required for the complete repression of $B m l p 3$ in the silk glands might be absent from the isolated 1,119 bp DNA fragment. A developmental analysis of DsRed expression detected low 
A
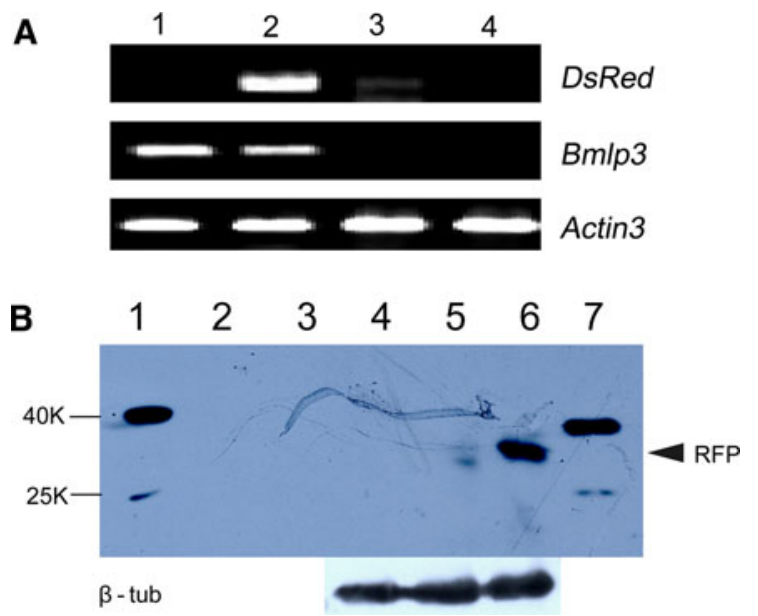

Fig. 4 Tissue-specific expression of DsRed in transgenic silkworm. a Monitoring the level of DsRed mRNA in the day7 fifth instar larvae by RT-PCR. Lanes 1-4 are RT-PCR results using cDNA template derived from RNA isolated from the fat body of wild type (1), transgenic (2) silkworms, and the silk glands (3) and midgut (4) of transgenic silkworms. b Monitoring the level of DsRed protein in the day-7 fifth instar larvae by Western blotting. Lanes $1-7$ contain a protein marker (1), $10 \mu \mathrm{g}$ of total protein from wild-type fat bodies (2) and hemolympth (3), the silk gland (4), midgut (5) and fat body (6) of transgenic larva and $1 \mu \mathrm{g}$ of recombinant RFP (7). The numbers on left side of gels indicate the molecular mass $(\mathrm{kDa})$. Arrowhead on the right indicates the recombinant RFP

levels of DsRed transcripts in day-3 fourth instar larvae through day-3 fifth instar larvae, DsRed transcript levels then increased with maximal expression occurring in the mid-pupal stage, and finally disappeared at day- 8 pupae. The accumulation of DsRed mRNA follows a pattern very similar to that observed for the transcripts of the endogenous Bmlp3 gene (Fig. 5a). Western blot analysis of DsRed protein expression confirmed the patterns of DsRed gene expression. However, the DsRed protein was only detected from day-5 fifth instar larvae and disappeared in day-9 pupae (Fig. 5b), suggesting that the DsRed protein might have been released into the hemolymph.

In addition, quantity of RFP in the fat body of transgenic silkworm is determined by densitometric measurement of the immunoblot using Quantity One software. It showed that each $10 \mu \mathrm{g}$ fat body of transgenic silkworm contains $0.54 \mu \mathrm{g}$ pure RFP. As we know proteins of fat body are synthesized abundantly and rapidly from late fifth instar silkworm larva to pupae, achieving about $30 \%$ of whole body (Xiang 2005). That is to say the pure RFP synthesized by fat body account for about $1.6 \%$ of single transgenic silkworm/pupae, suggesting the potential utility of the Bmlp3 promoter for production of recombinant proteins in the fat body of transgenic silkworm.

\section{Future work}

In this study, we report for the first time the isolation and characterization of a fat body-specific promoter from Bmlp3, a gene encoding a member of the silkworm $30 \mathrm{~K}$ protein family. Our results demonstrated that a $1,119 \mathrm{bp}$ fragment from the $5^{\prime}$ end of Bmlp3 had promoter activity sufficient to direct fat body-specific expression of DsRed reporter gene. This promoter-containing fragment results in useful temporal and spatial patterns of gene expression and is a useful tool for functionally analyzing interesting genes in the fat body of transgenic silkworms. Next, we will focus on identifying the key regulatory elements and putative transcription factors responsible for stageand tissue-specific expression of the Bmlp3 promoter using single base mutagenesis and/or promoter deletion strategy, to promote our better understanding of characters of the Bmlp3 promoter and even the synthesis and regulation of $30 \mathrm{~K}$ proteins.

As described above, the silkworm fat body is highly active in protein synthesis. Our results demonstrated the utility of the Bmlp3 promoter to produce recombinant proteins in the fat body of transgenic silkworm/ pupae, with an obvious advantage of requiring little need for protein purification. Silkworm pupae have been a favorite food in China from ancient times (Yang et al. 2009). In addition, it can also be processed directly into animal feeds and is a valuable and inexpensive nutrient source. The recombinant proteins produced in fat body cells of the transgenic silkworm/pupae are wrapped in a coating of fat and can avoid degradation after direct cooking or feeding to animals. In our future work we will test the feasibility of using the $B m l p 3$ promoter to regulate the expression of recombinant proteins such as antibiotics and animal vaccines in the fat body of transgenic silkworms. 


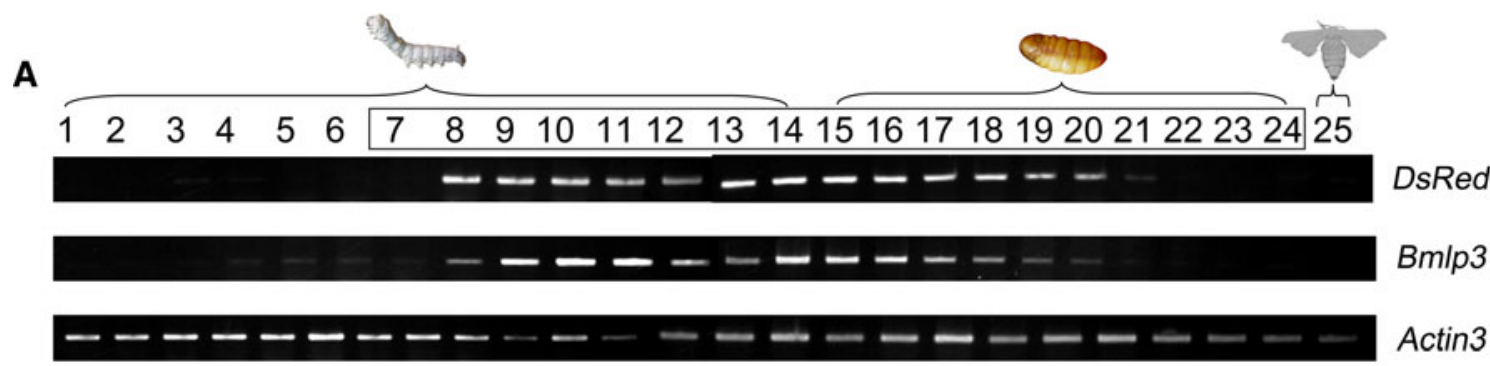

B

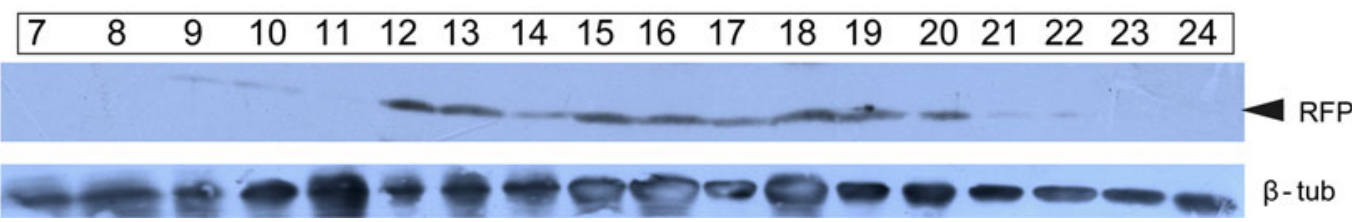

Fig. 5 Stage-specific expression of DsRed in transgenic silkworm. a Transcriptional level of DsRed at different stages of transgenic silkworm by RT-PCR. Lanes 1-25 are RT-PCR results using cDNA template derived from RNA isolated from day-1 fourth instar larvae (1), day-2 fourth instar larvae (2), day3 fourth instar larvae (3), molting stage of fourth instar larvae (4), day-1 fifth instar larvae (5), day-2 fifth instar larvae (6), day3 fifth instar larvae (7), day-4 fifth instar larvae (8), day-5 fifth instar larvae (9), day-6 fifth instar larvae (10), day-7 fifth instar larvae (11), day-1 after wandering (12), day-2 after wandering (13), day-3 after wandering (14), day-1 pupa (15), day-2 pupa

Acknowledgments We thank Professor Dr. Qingli Feng of South China Normal University, China, for providing the Spodoptera litura embryonic cell line Spli-221. We also thank Professor David O'Brochta of University of Maryland, USA, for improving this manuscript. This work was supported by the Grant (No. 31000981) from National Natural Science Foundation of China, Grant (No. 2012CB114600) from the National Basic Research Program of China, and Grant (No. kb2011008) from the Doctoral Innovation Fund of Southwest University.

Open Access This article is distributed under the terms of the Creative Commons Attribution License which permits any use, distribution, and reproduction in any medium, provided the original author(s) and the source are credited.

\section{References}

Cheng DJ, Xia QY, Zhao P, Wang ZL, Xu HF, Li GR, Lu C, Xiang ZH (2006) EST-based profiling and comparison of gene expression in the silkworm fat body during metamorphosis. Arch Insect Biochem Physiol 61:10-23

Delaney SJ, Smith DF, McClelland A, Sunkel C, Glover DM (1986) Sequence conservation around the $5^{\prime}$ ends of the larval serum protein 1 genes of Drosophila melanogaster. J Mol Biol 189:1-11
(16), day-3 pupa (17), day-4 pupa (18), day-5 pupa (19), day-6 pupa (20), day-7 pupa (21), day-8 pupa (22), day-9 pupa (23), day10 pupa (24), and moth (25). Gene-specific primers included those to detect the DsRed transgene (DsRed), endogenous Bmlp3 (Bmlp3) and Actin3 (Bmactin3). b Western blot analysis of DsRed protein expression in transgenic silkworms at different stages. Lanes 7-24 have proteins isolated from the same stages of those boxed lanes in (a). The filter was probed using an anti-RFP antibody (RFP) then stripped and re-probed using an anti $\beta$-tubulin antibody $(\beta$-tub)

Fujiwara Y, Yamashita O (1992) Gene structure of Bombyx mori larval serum protein $(B m L S P)$. Insect Mol Biol 1:63-69

Horn C, Wimmer EA (2000) A versatile vector set for animal transgenesis. Dev Genes Evol 210:630-637

Hou Y, Xia Q, Zhao P, Zou Y, Liu H, Guan J, Gong J, Xiang Z (2007) Studies on middle and posterior silk glands of silkworm (Bombyx mori) using two-dimensional electrophoresis and mass spectrometry. Insect Biochem Mol Biol 37(5): 486-496

Izumi S, Fujie J, Yamada S, Tomino S (1981) Molecular properties and biosynthesis of major plasma proteins in Bombyx mori. Biochim Biophys Acta 670:222-229

Izumi S, Kiguchi K, Tomino S (1984) Hormonal regulation of biosynthesis of major plasma proteins in Bombyx mori. Zool Sci 1:223-228

Matsumoto N, Nakanishi Y, Natori S (1986) Homologies of nucleotide sequences in the $5^{\prime}$-end regions of two developmentally regulated genes of Sarcophaga peregrina. Nucleic Acids Res 14:2685-2698

Mori S, Izumi S, Tomino S (1991a) Structures and organization of major plasma protein genes of the silkworm Bombyx mori. J Mol Biol 218:7-12

Mori S, Izumi S, Tomino S (1991b) Complete nucleotide sequences of major plasma protein genes of Bombyx mori. Biochim Biophys Acta 1090:129-132

Ogawa N, Kishimoto A, Asano T, Izumi S (2005) The homeodomain protein $\mathrm{PBX}$ participates in $\mathrm{JH}$-related suppressive 
regulation on the expression of major plasma protein genes in the silkworm, Bombyx mori. Insect Biochem Mol Biol 35:217-229

Sakai N, Mori S, Izumi S, Haino-Fukushima K, Ogura T, Maekawa H, Tomino S (1988) Structures and expression of mRNAs coding for major plasma proteins of Bombyx mori. Biochim Biophys Acta 949:224-232

Sakurai H, Fujii T, Izumi S, Tomino S (1988a) Structure and expression of gene coding for sex-specific storage protein of Bombyx mori. J Biol Chem 263:7876-7880

Sakurai H, Fujii T, Izumi S, Tomino S (1988b) Complete nucleotide sequence of gene for sex-specific storage protein of Bombyx mori. Nucleic Acids Res 16:7717-7718

Sun Q, Zhao P, Lin Y, Hou Y, Xia QY, Xiang ZH (2007) Analysis of the structure and expression of the $30 \mathrm{~K}$ protein genes in silkworm, Bombyx mori. Insect Sci 14:5-14

Tamura T, Thibert C, Royer C, Kanda T, Abraham E, Kamba M, Komoto N, Thomas JL, Mauchamp B, Chavancy G, Shirk P, Fraser M, Prudhomme JC, Couble P (2000) Germline transformation of the silkworm Bombyx mori L. using a piggyBac transposon-derived vector. Nat Biotechnol 18:81-84

Tojo S, Nagata M, Kobayashi M (1980) Storage protein in the silkworm, Bombyx mori. Insect Biochem 10:289-303

Tomino S (1985) Major plasma proteins of Bombyx mori. Zool Sci 2:293-303

Willott E, Wang XY, Wells MA (1989) cDNA and gene sequence of Manduca sexta arylphorin, an aromatic amino acid-rich larval serum protein. Homology to arthropod hemocyanins. J Biol Chem 264:19052-19059

Xia Q, Zhou Z, Lu C et al (2004) A draft sequence for the genome of the domesticated silkworm (Bombyx mori). Science 306:1937-1940

Xia Q, Cheng D, Duan J, Wang G, Cheng T, Zha X, Liu C, Zhao P, Dai F, Zhang Z, He N, Zhang L, Xiang Z (2007) Microarraybased gene expression profiles in multiple tissues of the domesticated silkworm, Bombyx mori. Genome Biol 8:R162

Xiang Z (2005) Biology of sericulture. China Forestry Publishing House, Beijing

Yang Y, Tang L, Tong L, Liu H (2009) Silkworms culture as a source of protein for humans in space. Adv Space Res 43:1236-1242

Zhang Y, Dong Z, Liu S, Yang Q, Zhao P, Xia Q (2012a) Identification of novel members reveals the structural and functional divergence of lepidopteran-specific Lipoprotein_11 family. Funct Integr Genomics 12(4):705-715

Zhang Y, Zhao P, Liu H, Dong Z, Yang Q, Wang D, Xia Q (2012b) The synthesis, transportation and degradation of BmLP3 and BmLP7, two highly homologous Bombyx mori 30K proteins. Insect Biochem Mol Biol 42(11):827-834

Zhao A, Zhao T, Zhang Y, Xia Q, Lu C, Zhou Z, Xiang Z, Nakagaki M (2010) New and highly efficient expression systems for expressing selectively foreign protein in the silk glands of transgenic silkworm. Transgenic Res 19:29-44 